\title{
Properties of Magnetosome Suspension under the Influence of Magnetic Field
}

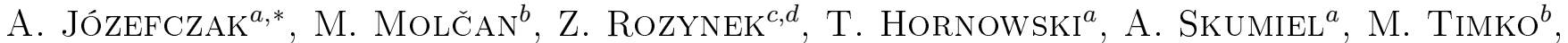 \\ J. TÓTHOVÁ ${ }^{e}$, P. KOPČANSKÝ ${ }^{b}$ AND B. LESZCZYŃSKI ${ }^{a, f}$ \\ ${ }^{a}$ Institute of Acoustics, Faculty of Physics, Adam Mickiewicz University, Umultowska 85, 61-614 Poznań, Poland \\ ${ }^{b}$ Institute of Experimental Physics, Slovak Academy of Sciences, Watsonova 47, 04001 Košice, Slovakia \\ ${ }^{c}$ Institute of Physical Chemistry, Polish Academy of Sciences, Kasprzaka 44/52, 01-224 Warszawa, Poland \\ ${ }^{d}$ Department of Physics, NTNU, Høgskoleringen 5, NO-7491 Trondheim, Norway \\ ${ }^{e}$ Department of Physics, Technical University of Košice, Park Komenského 2, 04200 Košice, Slovakia \\ ${ }^{f}$ NanoBioMedical Centre, Adam Mickiewicz University, Umultowska 85, 61-614 Poznań, Poland
}

\begin{abstract}
The magnetotactic bacteria synthesize chains of nanosized magnetic particles that function as a compass needle to navigate in the direction of the earth's magnetic field. Magnetosomes are bacterial magnetic nanoparticles containing iron mineral crystals of magnetite or greigite, enveloped by a natural biological membrane. The objective of the work is to study the influence of magnetic field on rheology and acoustic properties of magnetosome suspension. Experimental results show a clear effect of the external magnetic field on the acoustic parameters such as velocity and attenuation of ultrasonic wave, and the viscosity of the suspension, arising from the biogenic magnetic particles aggregation.
\end{abstract}

DOI: 10.12693/APhysPolA.127.629

PACS: 75.50.Mm, 43.35.Bf

\section{Introduction}

Nanosized magnetic particles have been studied extensively in recent years because of the potential for application in biomedicine. Chemically synthesized nanoparticles used in medical applications require good biocompatibility so appropriate modification of the particle surface is necessary. Biogenic magnetic particles such as bacterial magnetosomes, which are obtained from magnetotactic bacteria, are organelles consisting of magnetite $\left(\mathrm{Fe}_{3} \mathrm{O}_{4}\right)$ or greigite $\left(\mathrm{Fe}_{3} \mathrm{~S}_{4}\right)$ crystals enclosed by a biological membrane. Such membrane provides a high degree of biocompatibility without additional chemical treatment. The size of one magnetosome (i.e., magnetic particle with organic shell) is around $50 \mathrm{~nm}$ but they can join into chain-like structures. The chain of magnetosomes inside the bacteria provides a magnetic dipole which is used by the bacteria to swim along the earth's magnetic field line [1]. The magnetosomes are covered by biological material made of a majority of lipids and a minority of proteins. This biological coating results in negatively charged magnetosomes with a good dispersion in water [2]. Many authors review several medical applications of magnetosomes, such as those in magnetic drug delivery, magnetic resonance imaging (MRI), separate cells. Bacterial nanoparticles are also good candidates to carry out magnetic hyperthermia since they produce a large amount of heat when exposed to an alternating magnetic field [3].

* corresponding author; e-mail: aras@amu.edu.pl
Magnetosome suspension properties change under the influence of the magnetic field, similar to that of magnetic fluid. The objective of this work is to study the influence of magnetic field on rheology and acoustic properties of magnetosome suspension as part of its full description which is necessary before its application to humans [3].

\section{Sample preparation}

Bacterial magnetosomes were synthesized by the biomineralization process of magnetotactic bacteria Magnetospirillum strain AMB-1 under laboratory conditions. Bacteria produce magnetite $\left(\mathrm{Fe}_{3} \mathrm{O}_{4}\right)$ particles. The process of isolation of magnetosome chains (as shown in Fig. 1) from the body of bacteria consists of a series of cycles of sonication, centrifugation and magnetic decantation. The isolation parameters were as follows: (1) sonication for $5 \mathrm{~min},(2)$ centrifugation at $18,000 \mathrm{rpm}$ for $30 \mathrm{~min}$, and (3) magnetic decantation on permanent magnets FeNdB $(200 \mathrm{mT})$ which lasted $2 \mathrm{~h}$. The nonmagnetic part of the specimen was pipetted away and the sediment of magnetosome chains in the container was diluted with HEPES-EDTA (first 2 cycles), followed by washing with HEPES-NaCl (3rd cycle) and finally washing the samples with $10 \mathrm{mM}$ HEPES (about 10 cycles times in order to make the most perfect purification of the sample).

\section{Experimental results}

The ultrasound velocity and attenuation were measured by a resonance method using a ResoScan ${ }^{\mathrm{TM}}$ System (Germany) apparatus in which ultrasonic velocity is determined from a series of the resonance frequencies of the resonator cell recorded during initialization. Then, only a single resonance peak (chosen automatically) is 
tracked and from the changes of resonance frequency of this peak the ultrasonic velocity is evaluated. ResoScan ${ }^{T M}$ System permits the measurements of the ultrasonic velocity with the accuracy of $\pm 0.05 \mathrm{~m} / \mathrm{s}$ for low-volume samples $(0.200 \mathrm{ml})$. The ultrasound attenuation is evaluated on the basis of the slope of the phase of the resonance curve at the resonance frequency. The relative error of the ultrasonic attenuation coefficient is better than $5 \%$. Both ultrasound velocity and attenuation are measured for single wave frequency of $8 \mathrm{MHz}$.

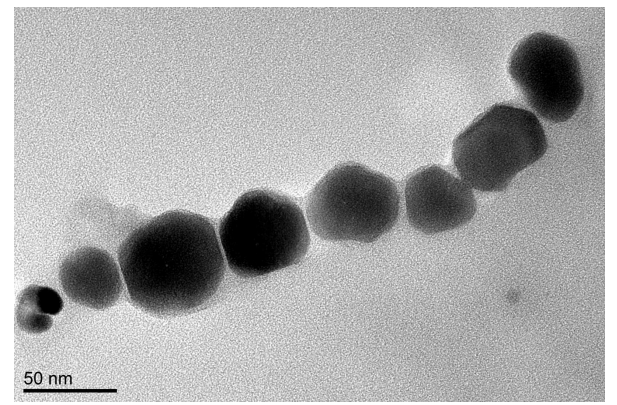

Fig. 1. Transmission electron image of magnetosome chain. Neighbouring biogenic magnetic particles in the chain are separated by small gaps filled with biological material.

First, the velocity of ultrasonic wave in magnetosome suspension was measured as a function of temperature in the absence of external magnetic field. Next, the sample was subjected to the magnetic field $H=300 \mathrm{kA} / \mathrm{m}$ for 30 min and the measurement of the acoustic parameters was repeated. Figure 2 presents the results. The ultrasound velocity in the suspension of bacterial magnetic particles is higher in comparison with that of carrier liquid. On the other hand, the ultrasound velocity in magnetic fluids with chemically synthesized $\mathrm{Fe}_{3} \mathrm{O}_{4}$ particles is smaller than that of carrier liquid $[4,5]$. The reason for this inverse behaviour is possibly due to the presence of natural biological membrane which surrounds magnetosomes [6].

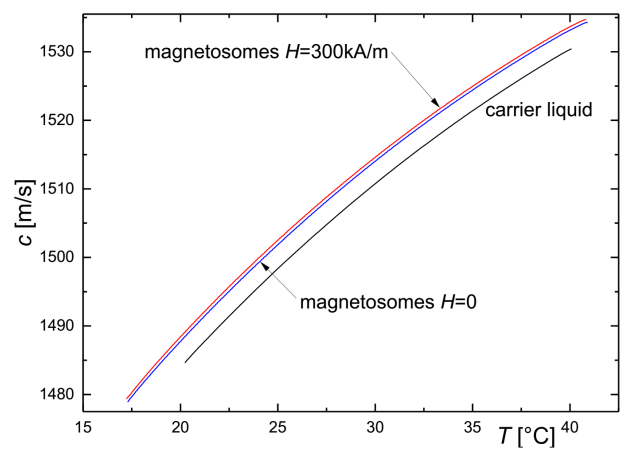

Fig. 2. The ultrasonic wave velocity $c$ as a function of temperature $T$ in carrier liquid and magnetosome suspension in both the absence and presence of the magnetic field.

The exposure of the sample to the magnetic field caused the change in ultrasonic wave velocity $(\Delta c=$ $0.65 \mathrm{~m} / \mathrm{s}$ ) determined after the field was turned off in comparison to that measured before the application of the field. This velocity hysteresis indicates magnetosomes aggregation. Though the attraction between the chains of magnetic particles is too weak to produce any aggregation of bacteria because the force between parallel bacterial magnetite backbones is strongly screened - the bacteria sense the geomagnetic field but not one another's compass [7] — but as the chains of magnetosomes are extracted from the bacteria to form an interacting system, the strength of the magnetostatic interactions between the chains of magnetosomes shows to increase [8]. Under the external force, i.e. strong magnetic field, the aggregation is generated by the interaction between the magnetosome chains. The chains of magnetosomes organize within wide bands and form a compact assembly. The chains attract each other, forming a long string of nanoparticles oriented in the direction of the magnetic field [9].

Figures 3 and 4 show a more detailed study of the influence of the magnetic field within the range of 50$300 \mathrm{kA} / \mathrm{m}$ on the ultrasound velocity and attenuation in magnetosome suspension. The results show that the magnetosomes aggregate. The aggregation is durable and the structure does not return to the initial state after the magnetic field has been removed.



Fig. 3. The change in ultrasonic wave velocity $c$ after the application of the magnetic fields.

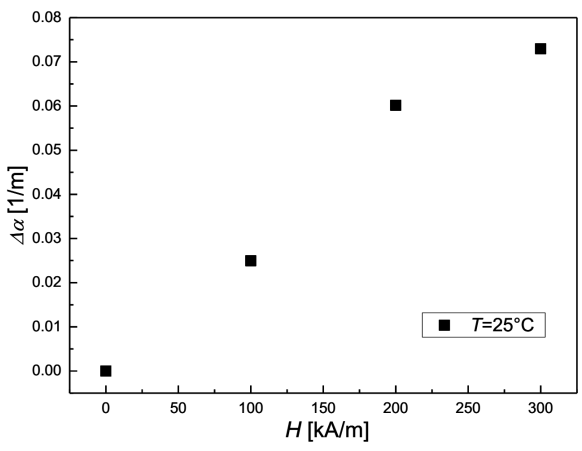

Fig. 4. The change in ultrasonic wave attenuation after the application of the magnetic fields.

The observed change in velocity and the attenuation of ultrasonic waves increase with the intensity of the magnetic field that was applied prior to the acoustic measurements. Thus the properties of the suspension depend on its magnetic history. Re-sonification is needed for the disintegration of aggregates. 
The measurements of viscoelastic properties also show the changing of magnetosome suspension structure induced by magnetic field because the viscoelastic properties are correlative to their microstructure. The magnetorheological measurements under the influence of magnetic field were carried out using a rotational rheometer Physica MCR300 (Anton Paar) equipped with a magnetorheological device MRD180.

It is a coaxial parallel plate-plate system with a diameter of $20 \mathrm{~mm}$ (PP 20/MRF) - Fig. 5. The distance between the plates was adjusted to $0.8 \mathrm{~mm}$. The built-in coil of the magnetorheological cell generated a magnetic field, $H$. During the measurement the field was oriented perpendicularly to the plates of the rheometer, and thus, perpendicularly to the direction of the flow.

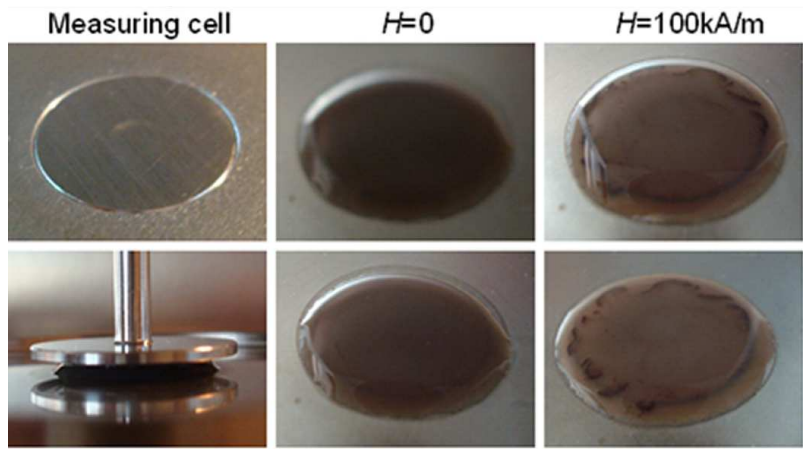

Fig. 5. The photographs of measurements cell (parallel plate-plate system) and drops of magnetosomes suspension without and after application of the magnetic field.

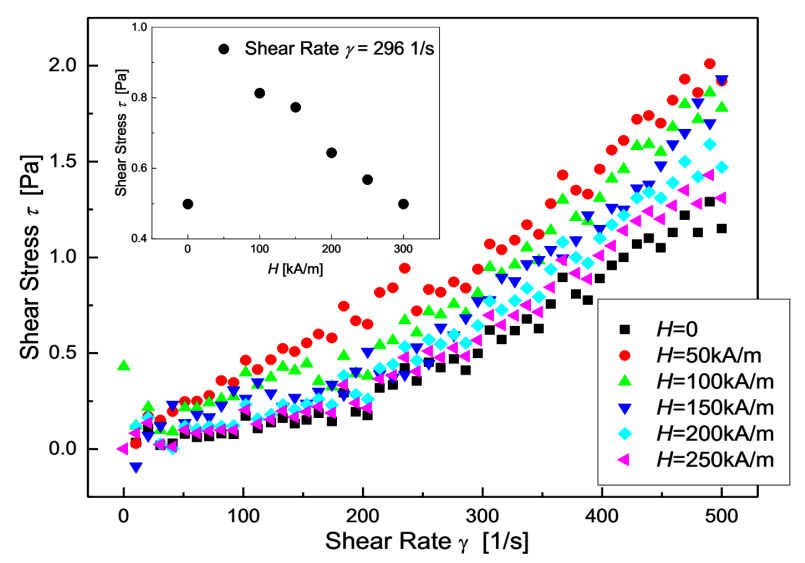

Fig. 6. Shear stress vs. shear rate for different values of magnetic field strength (the inset shows shear stress vs. magnetic field).

The measured shear stress $\tau$ vs. shear rate $\gamma$ for different values of magnetic field strength and without the presence of magnetic field is illustrated in Fig. 6 . It can be seen that magnetic field influences the viscosity $(\eta=\tau / \gamma)$ of magnetosomes suspension. Viscosity increases rapidly after applying the magnetic field, but later it decreases despite the increase of the magnetic field.
The dependence is explained in Fig. 5. The biogenic magnetic nanocolloid separates into two coexisting phases, as a result of the magnetically induced phase condensation [10]. The phase separation of the system into dense and dilute phases leads to the formation of discrete domain. When the shear strain increase the aggregates are pulled out from the gap on the outer edge of the plate despite the use of the plate-plate configuration of rheometer. That interferes with the measurement of viscosity. In the absence of a field the photographs show a homogeneous drop of the suspension, whereas after the application of magnetic field a clear separation of phases (condensation) can be noted on the circumference of the drop. The formation of aggregates leads to the appearance of viscoelastic effects or other non-Newtonian features in the magnetosome nanoparticle suspension in the presence of a magnetic field.

\section{Conclusions}

Magnetic field rearranges the magnetosomes leading to the formation of aggregate microstructures and the appearance of acoustic and viscoelastic properties of the magnetosome suspension. The experimental results show a clear effect of the external magnetic field on acoustic parameters. The velocity and the attenuation of the ultrasonic wave show a hysteresis which results from magnetosomes aggregation. The rheological properties of the studied magnetosome suspension also show the effect of the magnetic field.

\section{Acknowledgments}

This work was supported by Polish National Science Centre grant DEC-2011/03/B/ST7/00194 and by Projects Nos. 26110230097, 26110230061 and 26220220005 in the frame of Structural Funds of European Union. Z.R. acknowledges financial support from the Foundation for Polish Science through Homing Plus programme.

\section{References}

[1] D.A. Bazylinski, R.B. Frankel, Nat. Rev. Microbiol. 2, 217 (2004).

[2] E. Alphandéry, S. Faure, O. Seksek, F. Guyot, I. Chebbi, ACS Nano 5, 6279 (2011).

[3] E. Alphandery, I. Chebbi, F. Guyot, M. DurandDubief, Int. J. Hyperthermia 29, 801 (2013).

[4] M. Motozawa, Y. Iizuka, T. Sawada, J. Phys. Condens. Matter 20, 204117 (2008).

[5] A. Józefczak, A Skumiel, J. Magn. Magn. Mater. 323, 1509 (2011).

[6] A. Józefczak, A. Hashim, M. Molcan, T. Hornowski, A. Skumiel, M. Rajnak, P. Kopčanský, M. Timko, Magnetohydrodynamics 49, 411 (2013).

[7] A.P. Philipse, D. Maas, Langmuir 18, 9977 (2002).

[8] E. Alphandery, A.T. Ngo, C. Lefevre, I. Lisiecki, L.F. Wu, M.P. Pileni, J. Phys. Chem. C 112, 12304 (2008).

[9] E. Alphandery, Y. Ding, A.T. Ngo, Z.L. Wang, L.F. Wu, M.P. Pileni, ACS Nano 3, 1539 (2009).

[10] A. Józefczak, T. Hornowski, Z. Rozynek, A. Skumiel, J.O. Fossum, Int. J. Thermophys. 34, 609 (2013). 